\title{
Efficacy of Platelet-Rich Plasma for Bone Fusion in Transforaminal Lumbar Interbody Fusion
}

\author{
Go Kubota, Hiroto Kamoda, Sumihisa Orita, Kazuhidee Inage, Michihiro Ito, \\ Masaomi Yamashita, Takeo Furuya, Tsutomu Akazawa, Yasuhiro Shiga, Seiji Ohtori \\ Department of Orthopaedic Surgery, Graduate School of Medicine, Chiba University, Chiba, Japan
}

\begin{abstract}
Study Design: Retrospective case series.
Purpose: To examine the efficacy of platelet-rich plasma (PRP) for bone fusion in transforaminal lumbar interbody fusion (TLIF) using local bone grafting.

Overview of Literature: Several authors have reported the efficacy of PRP for bone union in animal models. However, the use of PRP for bone fusion in TLIF surgery has not been fully explored.

Methods: Twenty patients underwent single-level TLIF surgery because of L4 spondylolisthesis. An interbody fusion cage and local bone were used in nine patients (control group) and an interbody fusion cage, local bone, and PRP were used in 11 patients (PRP group). PRP was prepared from the patients' blood samples $(400 \mathrm{~mL}$ ) immediately before surgery. The duration of bone union and postoperative bone fusion rate were assessed using plain radiography at every 3 months postoperatively and computed tomography at 12 or 24 months postoperatively, respectively. Lower back pain, leg pain, and leg numbness were evaluated using the visual analog scale preoperatively and at $3,6,12$, and 24 months postoperatively.

Results: The platelet count was 8.7 times higher in PRP than in blood. The bone union rate was significantly superior in the PRP group than in the control group $(91 \%$ and $77 \%$, respectively; $p=0.035)$, whereas the average duration of bone union was not significantly different between the groups $(7.7 \pm 0.74$ and $10.0 \pm 2.00$ months, respectively; $p=0.131)$. There was no significant difference in lower back pain, leg pain, and leg numbness in both groups during follow-up $(p>0.05)$.

Conclusions Our study suggests that the use of PRP in TLIF surgery increases bone fusion rate.
\end{abstract}

Keywords: Spine; Platelet-rich plasma; Bone; Lumbar; Fusion

\section{Introduction}

The use of autologous iliac crest bone is the gold standard for spinal bone fusion; however, many authors have reported complications such as risk of infection, hematoma, fracture, wound healing problems, and donor site pain $[1,2]$. To avoid these complications, local bone, ceram- ics, demineralized bone matrix, and bone morphogenetic proteins have been used for lumbar fusion [3]. However, neck swelling, ectopic bone formation, radiculitis, and retrograde ejaculation have been reported with regard to bone morphogenetic proteins [4]. Furthermore, the use of bone morphogenetic proteins is not allowed in some Asian countries.

Received May 1, 2017; Revised May 30, 2017; Accepted Jun 8, 2017

Corresponding author: Seiji Ohtori

Department of Orthopaedic Surgery, Graduate School of Medicine, Chiba University, 1-8-1 Inohana, Chuo-ku, Chiba 260-8670, Japan

Tel: +81-43-226-2117, Fax: +81-43-226-2116, E-mail: sohtori@faculty.chiba-u.jp 
Platelet-rich plasma (PRP) has recently been used for muscle, tendon, and bone healing. Even a small amount of PRP contains a high concentration of platelets and osteoinductive autologous growth factors such as platelet-derived growth factor and transforming growth factor beta $[5,6]$. The use of PRP along with local bone for posterolateral lumbar fusion (PLF) enhanced bone fusion, and PRP combined with hydroxyapatite for posterior lumbar interbody fusion (PLIF) also enhanced bone fusion in a rat model $[7,8]$. Some authors have reported the use PRP for PLF or anterior or posterior interbody fusion; however, the results are controversial [9-15]. Another major issue is the lack of controlled clinical trials to evaluate how PRP increases the rate of spinal fusion and to what extent [16]. Furthermore, most studies have used PRP along with autologous iliac crest bone to achieve bone fusion [9-15].

The purpose of the present study was to examine the clinical efficacy of PRP for bone union after transforaminal lumbar interbody fusion (TLIF) surgery using local bone grafting.

\section{Materials and Methods}

\section{Patients}

The subjects of the present study included patients from our hospital diagnosed with lumbar spinal stenosis with L4 or L5 spondylolisthesis and instability between July 2009 and November 2015. Spinal instability was defined as $>5 \%$ of anterior translation of the vertebra and $>5^{\circ}$ of translocation between the flexed and extended positions on lumbar radiographic examination. Patients with more than two-level pathological lesions, past lumbar surgery, or other infectious or inflammatory diseases, such as spinal tumors, infection, and trauma, were excluded. In this retrospective case-control study, 20 patients underwent single-level TLIF surgery. An interbody fusion cage and local bone were used in nine patients (control) and an interbody fusion cage, local bone, and PRP were used in the other 11 patients (PRP group).

\section{PRP preparation}

Immediately before surgery, $400 \mathrm{~mL}$ of peripheral venous blood was taken from each patient for PRP preparation. The blood was processed using a two-stage centrifugation method (CR7B3; Hitachi Koki Co. Ltd., Tokyo, Japan).
The first centrifugation was performed at 1,660 rpm for 5 minutes. Consequently, the plasma was separated from the red blood cells. The second centrifugation was performed at 1,450 rpm for 15 minutes to pellet the platelets. The pelleted platelets were temporarily isolated from the supernatant platelet-poor plasma (PPP). To adjust the PRP volume, a portion of PPP was mixed with the pelleted platelets. Finally, $22 \mathrm{~mL}$ of PRP was generated, of which $2 \mathrm{~mL}$ was used to count the number of platelets using a hematology analyzer.

During surgery, $0.5 \mathrm{~mL}$ of $1,000 \mathrm{U} / \mathrm{mL}$ liquid thrombin solution (Mochida Pharmaceutical Co. Ltd., Tokyo, Japan) and $1 \mathrm{~mL}$ of $2 \%$ calcium chloride solution (Otsuka Pharmaceutical Co. Ltd., Tokyo, Japan) were added to the remaining $20 \mathrm{~mL}$ of PRP to activate the platelets and prepare a PRP gel mixed with the autogenous local bone graft for insertion into the interbody fusion cage. Red blood cells were returned to patients intravenously during surgery.

\section{Surgical technique}

Conventional single-level TLIF was performed using pedicle screws and local bone graft. No additional osteoconductive products were used for the spinal fusion. In the PRP group, $10 \mathrm{~mL}$ of activated PRP was added with local bone into the cage (PRP was not used in the control group). The same team of surgeons performed all surgical procedures in a similar manner using CAPSTONE (Medtronic Inc., Memphis, TN, USA) for the cage and CD Horizon Legacy (Medtronic Inc.) for the pedicle screw fixation systems.

\section{Outcome measurements}

1) Quantification of platelet count

After PRP preparation, platelets in whole blood and PRP were counted using a hematology analyzer (K-4500; Sysmex Corp., Kobe, Japan).

\section{2) Assessment of final bone union rate}

The final bone union rate and area of bone union between the L4 and L5 vertebrae were assessed at 12 or 24 months postoperatively using coronal-plane three-dimensional computed tomography $(\mathrm{CT})$ reconstruction. Bone union assessed using CT was defined as bridging bone remodeling occurring between the adjacent vertebrae. If cyst for- 
mation between the adjacent vertebrae and pedicle screw loosening was observed using CT, we defined this as non-union. Evaluation was performed by three surgeons blinded to the condition. Fusion was defined as at least two observers simultaneously detecting bone fusion.

In order to assess the reliability of the bone fusion, two independent observers classified each case twice within a 1-week interval to measure intra- and inter-observer differences. The weighted kappa coefficient $(\kappa)$ was calculated for each spine surgeon based on their first and second observations for within and between comparisons. $\kappa$ varies between 0 and 1 ; the greater the value of $\kappa$, the higher the agreement rate. A $\kappa$ value of $0-0.20$ indicates slight agreement, $0.21-0.40$ indicates fair agreement, $0.41-0.60$ indicates moderate agreement, $0.61-0.80$ indicates substantial perfect agreement, and $\geq 0.81$ is regard as an almost perfect agreement according to the interpretation by Landis and Koch [17].

\section{3) Assessment of duration of bone union}

Duration of bone union was assessed over a period of 24 months postoperatively using lateral flexion-extension radiographic images. Radiological findings were obtained every 3 months postoperatively. Radiographic spinal union was defined as $<1.0^{\circ}$ instability between the flexed and extended positions. The assessment of duration of bone union was blinded and performed by three different surgeons. The time to bone union was the period between surgery and when at least two observers simultaneously detected bone fusion.

\section{4) Clinical evaluation}

We evaluated the changes in lower back pain, leg pain, and leg numbness preoperatively and at 3, 6, 12, and 24 months postoperatively. To evaluate pain, the Visual Ana$\log$ Scale (VAS) scores ( 0 , no pain; 10 , worst pain) for low- er back pain, leg pain, and leg numbness were recorded and compared.

\section{Statistical analysis}

Student $t$-test with standard error of mean (SEM) was used to assess the statistical significance of data. SEM was calculated using the standard error of proportions. All data were statistically evaluated using IBM SPSS software ver. 19.0 (IBM Corp., Armonk, NY, USA). $p<0.05$ was considered significant.

\section{Results}

\section{Demographic data}

Patients' demographic data are shown in Table 1. There was no significant difference in the number of patients, sex, average age, and number of fixation levels between the control and PRP groups $(p>0.05)$.

\section{Platelet-rich plasma and blood serum platelet counts}

Mean \pm SEM blood serum platelet and PRP platelet counts were $25.3 \pm 2.94\left(\times 10^{4} / \mu \mathrm{L}\right)$ and $189.7 \pm 34.10\left(\times 10^{4} / \mu \mathrm{L}\right)$, respectively. The platelet count in PRP was 8.7 times higher than that in blood $(p<0.05)$ (Table 2).

\section{Assessment of final bone union rate and period to fu- sion}

The $\kappa$ values of the bone fusion for intra- and inter-observers ranged from 0.81 to 0.85 , indicating almost perfect agreement. Fig. 1 and Table 3 show the results of the radiographic and CT evaluations of spinal fusion. Two years postoperatively, CT showed that $77 \%$ of the segments ex-

Table 1. Demographic characteristics of patients

\begin{tabular}{lccc} 
Characteristic & Control & Platelet-rich plasma & $p$-value \\
No. of patients & 9 & 11 & 0.940 \\
Sex & & & \\
\hline Male & 4 & 5 & \\
Female & 5 & $59.4 \pm 3.85(30-75)$ & 0.253 \\
Age $(y r)$ & $63.3 \pm 4.23(33-75)$ & 1 level: $11(L 4 / 5=7, L 5 / S=4)$ & 0.136 \\
\hline No. of fixation levels: no. of patients & 1 level: $9(L 4 / 5=6, L 5 / S=3)$ & & \\
\hline
\end{tabular}

Values are presented as number or mean \pm standard error of mean (range). 
Table 2. Concentration of platelet

\begin{tabular}{lrrr} 
No. of patient & Platelet-rich plasma Plt $\left(\times 10^{4} \mu \mathrm{L}\right)$ & Serum Plt $\left(\times 10^{4} \mu \mathrm{L}\right)$ & \multicolumn{2}{c}{ Concentration } \\
\hline 1 & 263.0 & 32.2 & 8.2 \\
\hline 2 & 205.0 & 7.0 & 29.3 \\
\hline 3 & 129.0 & 31.1 & 4.1 \\
\hline 4 & 366.9 & 24.8 & 14.8 \\
\hline 5 & 399.5 & 43.1 & 9.3 \\
\hline 6 & 189.0 & 32.4 & 5.8 \\
\hline 7 & 109.0 & 27.6 & 3.9 \\
\hline 8 & 91.0 & 18.4 & 4.9 \\
\hline 9 & 39.6 & 16.6 & 2.4 \\
\hline 10 & 127.5 & 18.9 & 6.7 \\
\hline 11 & 167.0 & 25.8 & 6.5 \\
\hline Average \pm SEM & $189.7 \pm 34.10$ & $25.3 \pm 2.94$ & $8.7 \pm 2.29$ \\
\hline
\end{tabular}

PIt, platelet; SEM, standard error of mean.

Table 3. Evaluation of spinal fusion

\begin{tabular}{lccc} 
Variable & Control & Platelet-rich plasma & $p$-value \\
No. of fixation levels: no. of patients & 1 level: 9 & 1 level: 11 & 0.136 \\
Bone union rate, computed tomography (\%) & 77 & 91 & 0.035 \\
\hline Period until bone union (mo) & $10.0 \pm 2.00$ & $7.7 \pm 0.74$ & 0.131 \\
\hline
\end{tabular}

Values are presented as number or mean \pm standard error of mean.
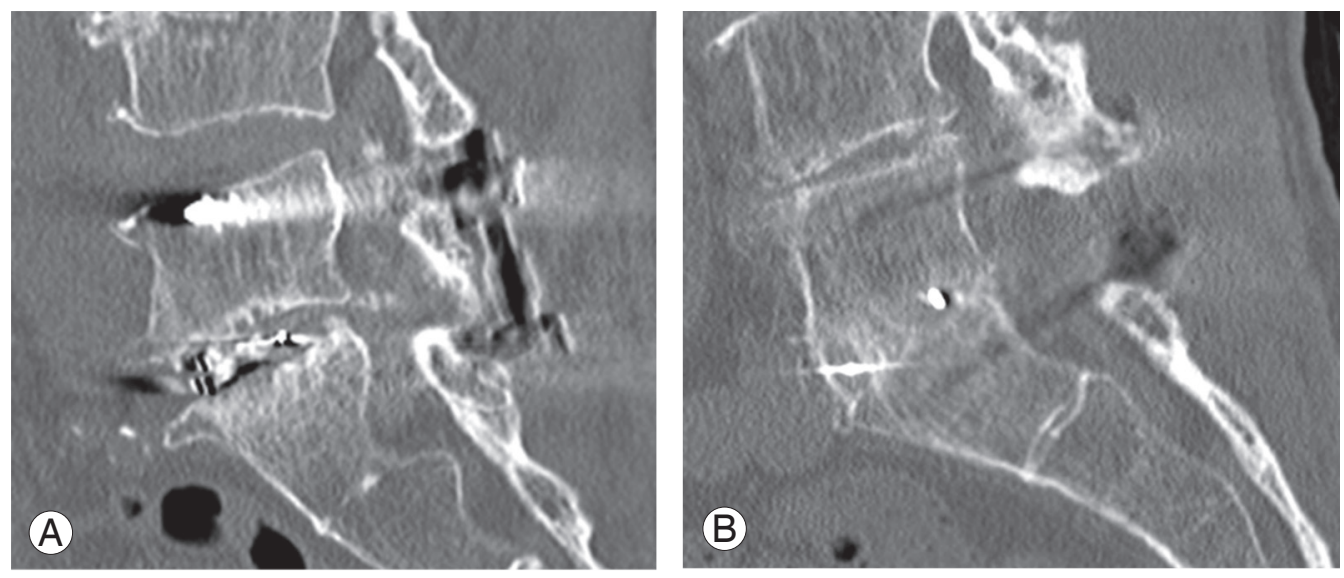

Fig. 1. Non-fusion case in the control group (A) and fusion case in the platelet-rich plasma group (B), as evaluated using computed tomography.

hibited fusion between the vertebrae in the control group, whereas $91 \%$ of the segments exhibited fusion in the PRP group. The rate of bone fusion in the PRP group was significantly greater than that in the control group $(p=0.035)$ (Table 3). The mean time required for bone union was $10.0 \pm 2.00$ and $7.7 \pm 0.74$ months in the control and PRP groups, respectively (Table 3). The mean time required for bone union tended to be shorter in the PRP group compared with the control group, but the fusion period was not significantly different between the groups $(p=0.131)$ (Table 3). 

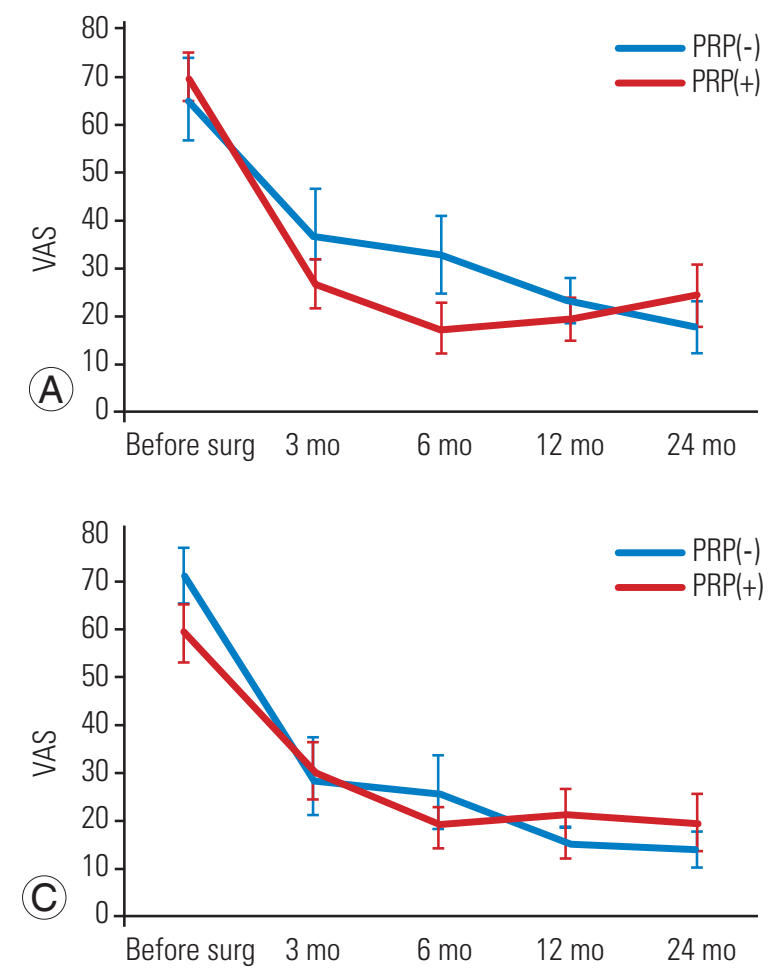

\section{Visual Analog Scale pain score}

The VAS scores for lower back pain, leg pain, and leg numbness were not significantly different between the two groups preoperatively ( $p>0.05)$ (Fig. 2). The three VAS scores significantly improved postoperatively compared with preoperatively in both groups $(p<0.05)$ (Fig. 2). However, during 24-month follow-up postoperatively, the three VAS scores were not significantly different between the two groups ( $p>0.05$ ) (Fig. 2).

\section{Discussion}

In the present study, addition of PRP in TLIF surgery promoted bone fusion during a 2-year follow-up period. However, the use of PRP did not significantly shorten the time to bone union nor was there any significant difference in lower back pain, leg pain, and leg numbness in either group during follow-up. The platelet count was higher in PRP than in blood. These results suggest that the use of PRP in TLIF surgery can significantly promote bone fusion, but it did not reduce the time required for bone union or pain postoperatively.

Regarding spinal surgery, Lowery et al. [11] first reported 19 cases of lumbar spinal fixation using platelet

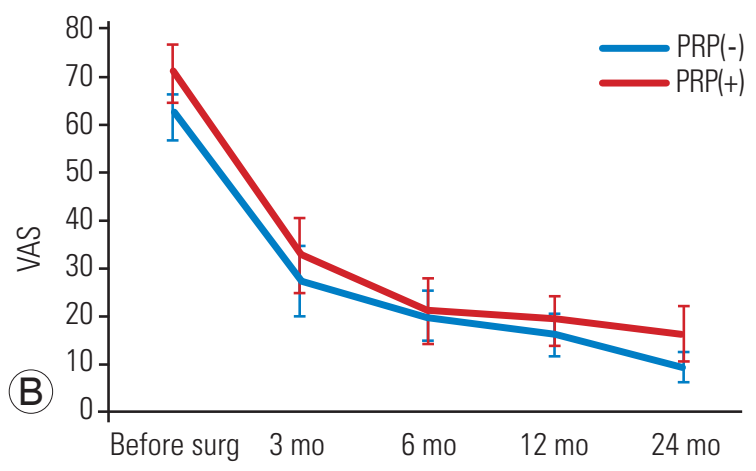

Fig. 2. VAS pain scores for lower back pain (A), leg pain (B), and leg numbness (C) were not significantly different between the two groups preoperatively $(p<0.05)$. Lower back pain at 6 months postoperatively was significantly better in the PRP group than in the control group ( $p=0.03)$; however, the VAS score for lower back pain except for 6 months, leg pain, and leg numbness were not significantly different between the two groups and at each time point postoperatively. Values are presented as mean \pm standard error of the mean. VAS, Visual Analog Scale; PRP, platelet-rich plasma.

concentrates (posterior fusion, $\mathrm{n}=15$; anterior intradiscal fusion, $n=4)$. In five patients, bone union was confirmed at the time of the second surgery, whereas in the remaining 14 patients, bone union was confirmed using radiographic assessment [11]. Meanwhile, Weiner and Walker [12] examined the fusion rate in single-level PLF using iliac crest bone graft or iliac crest bone graft plus platelet concentrates. They concluded that the use of platelet concentrates resulted in inferior fusion rates compared with autogenous bone graft alone [12]. Carreon et al. [13] compared 76 consecutive patients who underwent instrumented PLF with autologous iliac crest bone graft mixed with platelet concentrates with a control group who underwent the same surgery with autologous bone graft alone and concluded that platelet gel failed to enhance fusion rate. In the present study, the use of PRP for TLIF surgery improved fusion rate, which is not consistent with PLF surgery data. Previous authors used autologous iliac crest bone graft for fusion; however, we used local bone graft for fusion. In addition, we speculated that PRP added during PLF diffused to other areas and did not fully achieve fusion in PLF.

In contrast, several authors have reported the efficacy of PRP for interbody fusion surgery $[9,10]$. Twentythree patients who underwent TLIF with PRP application 
and a historical cohort (without PRP application) with a minimum of 2-year follow-up were compared [9]. Our study indicates that the platelet concentration of PRP was 4.8-fold higher than that of normal serum and that PRP can lead to faster fusion [9]. Thirty-seven patients who underwent anterior-posterior interbody fusion using iliac crest bone graft or allograft combined with PRP were evaluated for 2 years [10]. Radiographic results confirmed an $85 \%$ arthrodesis rate for the autograft patients, whereas PRP patients had an $89 \%$ fusion rate, indicating that PRP combined with an appropriate carrier is a reasonable alternative to autograft [10]. The results of the present study demonstrate that PRP along with autologous local bone better enhances the bone fusion rate compared with the control. A clinical and radiological trial in 40 patients showed that the use of PRP added to autograft iliac crest bone in PLIF did not lead to a superior fusion compared with autologous bone only [15]. In this study, the platelet concentration was unclear [15]. It has been suggested that at least five times the amount of platelets per cubic millimeter of peripheral blood is needed in the autologous platelet concentrate in order for it to be effective. It is also known that $95 \%$ of factors are released within the first hour, but platelets synthesize and secrete new factors for several days once the autologous platelet concentrate has been installed over the tissue to heal [18]. In the present study, the platelet count in PRP was 8.7 times higher than that in blood; thus, we speculate that the ideal platelet concentration in PRP should be $>5.0-9.0$ times more than that in the blood.

In the present study, despite the significant difference in fusion rate, there was no significant difference in VAS score between the two groups during follow-up. Some authors have reported a discrepancy between bone union and clinical results $[19,20]$. In lumbar posterior fusion surgery, successful fusion was not related to patient outcome [19], and pseudoarthrosis of the fusion mass was seen in $36 \%$ of patients postoperatively; however, clinical results were excellent because the development of fibrous union appeared to provide sufficient structural support [20]. Therefore, we conclude that our findings are supported by these earlier findings.

The present study has several limitations. First, the number of patients was small and this was a non-prospective randomized study. Second, the amount and quality of the local autograft were not uniform between patients, but this limitation existed in both treatment groups. Finally, we did not measure the concentration of growth factors in PRP. Further studies are required to support our hypothesis.

\section{Conclusions}

In conclusion, addition of PRP with a platelet count 8.7 times higher than that in the blood promoted bone fusion in TLIF surgery during a 2-year follow-up. However, use of PRP did not significantly shorten the time taken for bone union nor was there a significant difference in lower back pain, leg pain, and leg numbness in both groups during follow-up. These results suggest that the use of highly concentrated PRP in TLIF surgery can significantly promote bone fusion but does not reduce the time required for bone union or the pain postoperatively.

\section{Conflict of Interest}

No potential conflict of interest relevant to this article was reported.

\section{References}

1. Kurz LT, Garfin SR, Booth RE Jr. Harvesting autogenous iliac bone grafts: a review of complications and techniques. Spine (Phila Pa 1976) 1989;14:1324-31.

2. Sasso RC, LeHuec JC, Shaffrey C; Spine Interbody Research Group. Iliac crest bone graft donor site pain after anterior lumbar interbody fusion: a prospective patient satisfaction outcome assessment. J Spinal Disord Tech 2005;18 Suppl:S77-81.

3. Miyazaki M, Tsumura H, Wang JC, Alanay A. An update on bone substitutes for spinal fusion. Eur Spine J 2009;18:783-99.

4. McGovern SC, Fong W, Wang JC. Can bone morphogenetic protein binding peptide increase efficiency of bone formation? Spine (Phila Pa 1976) 2010;35:16559.

5. Noda M, Camilliere JJ. In vivo stimulation of bone formation by transforming growth factor-beta. Endocrinology 1989;124:2991-4.

6. Canalis E, McCarthy TL, Centrella M. Effects of platelet-derived growth factor on bone formation in vitro. J Cell Physiol 1989;140:530-7.

7. Kamoda H, Ohtori S, Ishikawa T, et al. The effect of platelet-rich plasma on posterolateral lumbar fusion 
in a rat model. J Bone Joint Surg Am 2013;95:110916.

8. Kamoda H, Yamashita M, Ishikawa T, et al. Plateletrich plasma combined with hydroxyapatite for lumbar interbody fusion promoted bone formation and decreased an inflammatory pain neuropeptide in rats. Spine (Phila Pa 1976) 2012;37:1727-33.

9. Hee HT, Majd ME, Holt RT, Myers L. Do autologous growth factors enhance transforaminal lumbar interbody fusion? Eur Spine J 2003;12:400-7.

10. Jenis LG, Banco RJ, Kwon B. A prospective study of Autologous Growth Factors (AGF) in lumbar interbody fusion. Spine J 2006;6:14-20.

11. Lowery GL, Kulkarni S, Pennisi AE. Use of autologous growth factors in lumbar spinal fusion. Bone 1999;25(2 Suppl):47S-50S.

12. Weiner BK, Walker M. Efficacy of autologous growth factors in lumbar intertransverse fusions. Spine (Phila Pa 1976) 2003;28:1968-70.

13. Carreon LY, Glassman SD, Anekstein Y, Puno RM. Platelet gel (AGF) fails to increase fusion rates in instrumented posterolateral fusions. Spine (Phila $\mathrm{Pa}$ 1976) 2005;30:E243-6.

14. Acebal-Cortina G, Suarez-Suarez MA, GarciaMenendez C, Moro-Barrero L, Iglesias-Colao R, Torres-Perez A. Evaluation of autologous platelet concentrate for intertransverse lumbar fusion. Eur Spine J 2011;20 Suppl 3:361-6.
15. Sys J, Weyler J, Van Der Zijden T, Parizel P, Michielsen J. Platelet-rich plasma in mono-segmental posterior lumbar interbody fusion. Eur Spine J 2011;20:1650-7.

16. Vaccaro AR, Sharan AD, Tuan RS, et al. The use of biologic materials in spinal fusion. Orthopedics 2001;24:191-7; quiz 198-9.

17. Landis JR, Koch GG. The measurement of observer agreement for categorical data. Biometrics 1977;33:159-74.

18. Alsousou J, Thompson M, Hulley P, Noble A, Willett $\mathrm{K}$. The biology of platelet-rich plasma and its application in trauma and orthopaedic surgery: a review of the literature. J Bone Joint Surg Br 2009;91:987-6.

19. Fischgrund JS, Mackay M, Herkowitz HN, Brower R, Montgomery DM, Kurz LT. 1997 Volvo Award winner in clinical studies: degenerative lumbar spondylolisthesis with spinal stenosis: a prospective, randomized study comparing decompressive laminectomy and arthrodesis with and without spinal instrumentation. Spine (Phila Pa 1976) 1997;22:280712.

20. Herkowitz HN, Kurz LT. Degenerative lumbar spondylolisthesis with spinal stenosis: a prospective study comparing decompression with decompression and intertransverse process arthrodesis. J Bone Joint Surg Am 1991;73:802-8. 